\title{
GPS driving: a digital biomarker for preclinical Alzheimer disease
}

\author{
Sayeh Bayat ${ }^{1,2^{*}}$ (D), Ganesh M. Babulal ${ }^{3,4,5}$, Suzanne E. Schindler ${ }^{3,4}$, Anne M. Fagan ${ }^{3,4,6}$, John C. Morris $3,4,6,7,8,9$,
} Alex Mihailidis ${ }^{1,2,10}$ and Catherine M. Roe ${ }^{3,4}$

\begin{abstract}
Background: Alzheimer disease (AD) is the most common cause of dementia. Preclinical AD is the period during which early $A D$ brain changes are present but cognitive symptoms have not yet manifest. The presence of $A D$ brain changes can be ascertained by molecular biomarkers obtained via imaging and lumbar puncture. However, the use of these methods is limited by cost, acceptability, and availability. The preclinical stage of AD may have a subtle functional signature, which can impact complex behaviours such as driving. The objective of the present study was to evaluate the ability of in-vehicle GPS data loggers to distinguish cognitively normal older drivers with preclinical AD from those without preclinical AD using machine learning methods.
\end{abstract}

Methods: We followed naturalistic driving in cognitively normal older drivers for 1 year with a commercial invehicle GPS data logger. The cohort included $n=64$ individuals with and $n=75$ without preclinical $A D$, as determined by cerebrospinal fluid biomarkers. Four Random Forest (RF) models were trained to detect preclinical AD. RF Gini index was used to identify the strongest predictors of preclinical AD.

Results: The F1 score of the RF models for identifying preclinical AD was 0.85 using APOE $\varepsilon 4$ status and age only, 0.82 using GPS-based driving indicators only, 0.88 using age and driving indicators, and 0.91 using age, APOE $\varepsilon 4$ status, and driving. The area under the receiver operating curve for the final model was 0.96 .

Conclusion: The findings suggest that GPS driving may serve as an effective and accurate digital biomarker for identifying preclinical AD among older adults.

Keywords: Naturalistic driving, Preclinical Alzheimer disease, Global positioning system, Machine learning

\section{Introduction}

Worldwide, around 50 million individuals are living with dementia, and this number is projected to increase to 152 million by 2050 [1]. Alzheimer disease (AD) is the most common form of dementia, accounting for 60 to $80 \%$ of cases [2-4]. Symptomatic AD impairs the cognitive and functional abilities required for performing activities of daily living, which can lead to hospitalizations, home care, and even institutionalization [5]. As a result,

\footnotetext{
* Correspondence: sayeh.bayat@mail.utoronto.ca

${ }^{1}$ Institute of Biomedical Engineering, University of Toronto, 550 University Avenue, Toronto, ON M5G 1X5, Canada

${ }^{2}$ KITE Research Institute, Toronto Rehabilitation Institute, Toronto, ON, Canada Full list of author information is available at the end of the article
}

$\mathrm{AD}$ can place significant financial and emotional burdens on family members and society at large [6]. Given the growing socioeconomic impacts of $\mathrm{AD}$, many studies have focused on the development of specific treatment strategies aimed at slowing down or even preventing the onset of symptomatic $\mathrm{AD}[7,8]$. However, these strategies may require $\mathrm{AD}$ to be diagnosed at an early stage before significant damage to the brain has occurred, when patients are still cognitively normal. Currently, the presence of $\mathrm{AD}$ brain pathology can be determined by molecular biomarkers obtained via imaging or lumbar puncture. However, the use of these methods is limited by cost, acceptability (i.e. willingness to participate in

(c) The Author(s). 2021 Open Access This article is licensed under a Creative Commons Attribution 4.0 International License, which permits use, sharing, adaptation, distribution and reproduction in any medium or format, as long as you give appropriate credit to the original author(s) and the source, provide a link to the Creative Commons licence, and indicate if changes were made. The images or other third party material in this article are included in the article's Creative Commons. licence, unless indicated otherwise in a credit line to the material. If material is not included in the article's Creative Commons licence and your intended use is not permitted by statutory regulation or exceeds the permitted use, you will need to obtain permission directly from the copyright holder. To view a copy of this licence, visit http://creativecommons.org/licenses/by/4.0/ The Creative Commons Public Domain Dedication waiver (http://creativecommons.org/publicdomain/zero/1.0/) applies to the data made available in this article, unless otherwise stated in a credit line to the data. 
research or assessments involving cerebrospinal fluid (CSF) collection), and availability [9, 10]. Therefore, there is an increasing need for low-cost and low-burden methods to make an early diagnosis of AD.

Preclinical $A D$ is the period during which early $A D$ brain changes are present but cognitive symptoms have not yet manifest [11]. The preclinical phase of $A D$ can include subtle cognitive changes, which may go unnoticed. However, emerging evidence suggests that these changes impact complex behaviours that involve both cognitive and functional abilities such as spatial navigation and driving. Since subtle cognitive changes may precede a clinical diagnosis of dementia by up to 20 years, tracking and recording navigational and driving abilities could potentially enable earlier identification of individuals with $\mathrm{AD}[12,13]$. A few studies have explored the utility of spatial navigation deficits as a marker for early and preclinical AD and discussed its high specificity for identifying at-risk individuals [13, 14]. This work, however, focuses on the potential of driving, the primary means of transportation for older adults, to detect underlying pathophysiology in preclinical AD.

Road tests and driving simulators are commonly used for assessing fitness-to-drive in clinical and healthy populations [15]. These standardized assessments, however, only measure performance in site-specific controlled conditions and do not assess daily driving behaviour in naturalistic settings. In addition, these assessment methods are methodologically limited by objectivity, availability, generalizability, and cost-effectiveness [15, 16]. To overcome these limitations, the field of driving research has shifted to collecting naturalistic outcomes using global positioning system (GPS) devices that can be installed in a participant's personal vehicle.

Several studies have adopted a naturalistic approach and showed that everyday driving behaviour is associated with symptomatic AD. These studies show that drivers with symptomatic $\mathrm{AD}$ are more likely to drive shorter distances, visit fewer unique destinations, and have a smaller driving space compared to cognitively intact drivers $[17,18]$. However, only a few studies, to date, have explored the impact of preclinical $\mathrm{AD}$ on driving behaviour. To our knowledge, our group is the first to use GPS to assess naturalistic driving behaviour among older drivers with preclinical AD [19]. In earlier work, we reported findings on a cross-sectional study that examined driving behaviour in a small sample $(n=20)$ of cognitively intact drivers with and without preclinical $\mathrm{AD}$. Later, we reported results of the extension of the data collection and evaluated driving behaviour changes over a 2.5-year period [20]. Most recently, we presented findings of a proof-of-concept study showing that preclinical $\mathrm{AD}$ can be identified by evaluating driving behaviours [21]. In discussing the results of that study, we noted that future research aimed at using everyday driving as a behavioural marker of AD should explore additional statistical modelling techniques to determine optimal combinations of variables [21].

The objective of this paper is to use machine learning techniques to test the ability of GPS data to distinguish persons with and without preclinical $\mathrm{AD}$, defined using cerebrospinal fluid, in a cohort of cognitively intact older adults from a longitudinal driving study. Specifically, we use machine learning to investigate the driving performance and driving space of older adults with and without preclinical AD. Further, we use feature selection to identify indicators that were the strongest discriminators of preclinical AD. This data-driven approach provides a foundation for the development of a novel neurobehavioural biomarker of $\mathrm{AD}$.

\section{Methods \\ Participants}

Participants were enrolled in longitudinal studies on ageing and dementia conducted at the Washington University Knight Alzheimer Disease Research Center and in a longitudinal driving study (R01AG056466). Participants who met the following criteria were included in the study: (1) were age 65 years or older, (2) had normal cognition at a clinical assessment that included assignment of the Clinical Dementia Rating ${ }^{\mathrm{Tm}}\left(\mathrm{CDR}^{\mathrm{Tw}}\right)$ [22], (3) underwent CSF collection, (4) possessed a valid driving licence, and (5) drove at least weekly, on average. Participants provided written informed consent, and all study procedures were approved by the Washington University Human Research Protection Office.

\section{CSF biomarkers and $A P O E$ genotyping}

CSF was collected as previously described [23]. CSF $A \beta 42$ and $A \beta 40$ analytes were measured using the Lumipulse G1200 automated assay system (Fujirebio, Malvern, PA). A $\beta 42 / A \beta 40<0.0673$ is highly concordant with positive status by amyloid positron emission tomography (PET) [24] and was used to identify individuals with preclinical AD. Taqman genotyping of rs7412 and rs429358 was used to determine APOE genotype [25].

\section{GPS data collection}

A GPS data logger (G2 Tracking Device ${ }^{\mathrm{T \mu}}$, Azuga Inc, San Jose, CA) was installed into the onboard diagnostics-II (OBD-II) port of each participant's vehicle. This data logger, together with custom software, comprises the Driving Real-World In-Vehicle Evaluation System (DRIVES) [20, 21, 26]. The DRIVES recorded date, time, latitude and longitude coordinates, and speed every $30 \mathrm{~s}$ when a vehicle was driven. For each participant, 1 
year of GPS driving data from January 1, 2019, to December 31, 2019, was included for analysis in this study. The 1-year study period was selected to account for seasonal variability in travel behaviours.

\section{GPS-based driving behaviour measures}

A comprehensive set of GPS-based indicators that describe everyday driving behaviour were examined. The indicators describe either the driving performance or driving space for each participant. Driving performance indicators capture speed, acceleration, and vehicle jerk characteristics, as well as aggressive driving incidents (e.g. hard braking), while driving space indicators capture the spatiotemporal characteristics of outdoor excursions. To select these indicators, we searched the literature for articles that used GPS technology to evaluate driving performance and measure life-space [20, 27, 28]. We selected the indicators that were most frequently used in these articles. The definitions of our proposed GPS-based indicators are presented in Table 1.

\section{Machine learning and statistical analyses}

All analyses were performed in Python. Data from participants with CSF biomarkers within 2 years of January 1, 2019, were selected. We regarded the prediction model for preclinical $\mathrm{AD}$ as a machine learning problem with a binary output, where class 0 included participants without preclinical $\mathrm{AD}(\mathrm{CSF} A \beta 42 / \mathrm{A} \beta 40 \geq 0.0673)$ and class 1 included participants with preclinical AD (CSF A $\beta 42 / A \beta 40<0.0673$ ). Random Forest (RF) classifier, a robust tree-structured machine learning algorithm, was used for this problem. RFs were selected because they have proven to outperform classical machine learning models in terms of accuracy and are more interpretable than deep learning models [32]. In addition, they are effective at handling high-dimensional data and are robust to outliers [33]. We trained four RF-based models with four sets of input variables: (1) age and APOE $\varepsilon 4$ status (carrier or non-carrier), (2) driving features only, (3) driving features and age, and (4) driving features, age, and $A P O E \& 4$ status. Predictive features of preclinical $\mathrm{AD}$ were ranked according to importance using RF Gini index, which is a method that ranks features based on

Table 1 Description of the GPS-based driving indicators

\begin{tabular}{|c|c|c|c|}
\hline Characteristics & Indicator & Abbreviation & Description \\
\hline \multirow[t]{7}{*}{ Driving space } & Average trip distance & TripDist & $\begin{array}{l}\text { The average distance travelled in each trip. TripDist is computed by taking the average of } \\
\text { all the trips that a participant has made during the study period. }\end{array}$ \\
\hline & Total travelled distance & TotalDist & The total distance travelled during the study period. \\
\hline & Number of trips & nTrips & $\begin{array}{l}\text { The total number of trips made during the study period. The trips are also placed into } \\
\text { five subgroups: (1) trips with a distance smaller than } 1 \mathrm{mi} \text {, (2) trips with a distance } \\
\text { between } 1 \text { and } 5 \mathrm{mi} \text {, (3) trips with a distance between } 5 \text { and } 10 \mathrm{mi} \text {, (4) trips with a } \\
\text { distance between } 10 \text { and } 20 \mathrm{mi} \text {, and (4) trips with a distance of more than } 20 \text { mi. }\end{array}$ \\
\hline & Radius of gyration & $\mathrm{Rg}$ & $\begin{array}{l}\text { Typical distance travelled by an individual, computed using [29]: } \\
r_{g}=\sqrt{\frac{1}{N} \sum_{i \in L} n_{i}\left(r_{i}-r_{c m}\right)^{2}} \\
\text { where } L \text { is the set of destinations by the individual, } r_{i} \text { is the latitude and longitude } \\
\text { coordinates of location } i_{,} n_{i} \text { is the visitation frequency of location } i, N=\sum_{i} n_{i} \text { is the total } \\
\text { number of visits of the individual, and } r_{c m} \text { is the center of mass of the visited destinations. }\end{array}$ \\
\hline & Entropy & $S$ & $\begin{array}{l}\text { The degree to which a participant's trip destinations are random (i.e. unpredictable) in } \\
\text { space and time [30]. S is assessed over the entire period of the study. }\end{array}$ \\
\hline & Number of night trips & nNightTrip & The average number of trips made after sunset. \\
\hline & $\begin{array}{l}\text { Number of unique } \\
\text { destinations }\end{array}$ & nUniqDest & $\begin{array}{l}\text { The total number of distinct destinations that an individual visited during the entire study } \\
\text { period. }\end{array}$ \\
\hline \multirow[t]{7}{*}{$\begin{array}{l}\text { Driving } \\
\text { performance }\end{array}$} & $\begin{array}{l}\text { Number of hard brakes } \\
\text { per mile }\end{array}$ & nHardBrake & $\begin{array}{l}\text { The average number of events with a deceleration rate of above } 8 \text { miles per hour in } 1 \mathrm{~s} \\
\text { per mile. }\end{array}$ \\
\hline & $\begin{array}{l}\text { Number of sudden } \\
\text { acceleration per mile }\end{array}$ & nSuddenAcc & $\begin{array}{l}\text { The average number of events with an acceleration rate of above } 8 \text { miles per hour in } 1 \mathrm{~s} \\
\text { per mile. }\end{array}$ \\
\hline & Overspeed & OverV & $\begin{array}{l}\text { The average number of trips with a speed of } 6 \text { miles per hour above the posted speed } \\
\text { limit. }\end{array}$ \\
\hline & Underspeed & UnderV & $\begin{array}{l}\text { The average number of trips with a speed of } 6 \text { miles per hour below the posted speed } \\
\text { limit. }\end{array}$ \\
\hline & Average speed & avg $\vee$ & The average speed of trips. \\
\hline & Average acceleration & $\operatorname{avgA}$ & The average acceleration of trips. \\
\hline & Average jerk & avgJ & $\begin{array}{l}\text { The average jerk of trips. Jerk is the rate of change of acceleration [31]; that is, more } \\
\text { abrupt brake actions or accelerations lead to higher jerk values. }\end{array}$ \\
\hline
\end{tabular}


how much they contribute to the model. All models were trained on $70 \%$ of the data and tested on the remaining $30 \%$ of the data. Note that each month record for each participant was considered an independent data point. To achieve the best performance, the models' specifications were selected based on incremental hyperparameter tuning; that is, each model was trained on many hyperparameters on training data, and then models that were performing better were selected. For performance evaluation, the precision, recall, and F1 score were calculated and compared across the four models. In this problem, we define preclinical $A D$ to be positive class and no preclinical $\mathrm{AD}$ to be negative class. Given these definitions, precision measures the number of true positives (i.e. preclinical AD) divided by the total number of predicted positives. That is, the ratio of predicted preclinical $\mathrm{AD}$ subjects who truly have preclinical $\mathrm{AD}$ over all subjects predicted to have preclinical AD. Recall, however, measures the ratio of true-positive cases over the total number of true-positive and false-negative cases. Finally, to compare the performances of the models, the F1 score is used, which combines precision and recall into a single number by taking their harmonic. In addition, in a similar approach to previous studies that tested the ability of a novel biomarker to distinguish between clinical groups [34, 35], a receiver operating curve (ROC) was generated and its area under the curve (AUC) was computed for each model. Models' performance metrics are reported on the test set, and 1000 bootstrapped samples were used to calculate $95 \%$ confidence intervals.

\section{Results}

\section{Sample characteristics}

Participant characteristics are presented in Table 2. The two groups did not differ significantly with regard to sex, race, or years of education level. The descriptive statistics and effect sizes of driving indicators are tabulated in Table 3.

\section{RF models}

The precision, recall, and F1 score for each model are presented in Table 4. The performance of the model for predicting preclinical $\mathrm{AD}$ improved with the addition of age alone, and age and APOE4 $\varepsilon 4$ status. The final model with all the features achieved an F1 score of 0.91 (95\% CI $0.893-0.937)$. The model was correct in identifying 96\% of individuals with preclinical AD (by the precision measure). Among all participants with preclinical AD, the model correctly identified $88 \%$ (by the recall measure).

The ROC area under the curve (AUC) for predicting preclinical AD from driving features was 0.82 (95\% CI 0.782-0.932) (Fig. 1) and improved with the addition of age alone to 0.94 (95\% CI $0.881-0.962)$, and age and APOE \&4 status 0.96 (95\% CI 0.0.903-0.981) (Fig. 1).

\section{Driving indicator importance}

The ranked importance scores of the features are presented in Fig. 2. The five most important features were APOE4 $\varepsilon 4$ status, age, average jerk, number of night trips, and radius of gyration.

\section{Discussion/conclusion}

Our findings suggest that driving may serve as an effective and accurate digital biomarker for identifying preclinical $\mathrm{AD}$ among older adults. In fact, the increasing availability of GPS devices is creating an environment where the naturalistic driving behaviours of older drivers are continuously monitored. This trend may make the analysis of naturalistic driving a non-invasive, unobtrusive, and low-cost solution for identifying individuals who are likely to have preclinical AD.

A novel finding of our study is the application of machine learning methods to a large dataset of GPS driving trajectories to predict preclinical AD. To provide an inclusive view of the driving behaviours of older adults with and without preclinical $\mathrm{AD}$, we created a comprehensive set of GPS-based indicators describing daily driving performance and space. Using driving indicators alone, our predictive model achieved an average F1 score of 0.82 (95\% CI $0.79-0.84$ ), indicating the model's high robustness and precision. The 0.89 precision score indicates that the model correctly predicts preclinical $\mathrm{AD}$ $89 \%$ of the time. The 0.76 recall score indicates that among the participants with preclinical $\mathrm{AD}$, the model correctly identified $76 \%$. Furthermore, compared to this model, the model with age and APOE $\varepsilon 4$ status alone

Table 2 Sample characteristics

\begin{tabular}{lll}
\hline & Without preclinical AD $(\boldsymbol{n}=\mathbf{7 5})$ & With preclinical AD $(\boldsymbol{n}=\mathbf{6 4})$ \\
\hline Age, years & $75.7 \pm 4.8$ & $79.1 \pm 4.90$ \\
APOE $\varepsilon 4+$ carrier, \% & 30 & 33 \\
Education, years & $16.4 \pm 2.3$ & $16.5 \pm 2.43$ \\
Sex, \% female & $51 \%$ & $47 \%$ \\
Race ${ }^{\text {a }}$ \% White & $84 \%$ & $92 \%$ \\
\hline
\end{tabular}

${ }^{\mathrm{a}}$ The sample includes only Blacks and Whites 
Table 3 Driving indicators' descriptive statistics and effect sizes using Cohen's d

\begin{tabular}{llll}
\hline & Without preclinical AD $(\boldsymbol{n}=\mathbf{7 5})$ & With preclinical AD $(\boldsymbol{n}=\mathbf{6 4})$ & ${\text { Cohen's } \mathbf{d}^{\mathbf{a}}}$ \\
\hline TripDist, $\mathbf{k m}$ & $8.1 \pm 2.6$ & $7.9 \pm 2.8$ & 0.07 \\
TotalDist, km & $891.5 \pm 371.4$ & $787.5 \pm 368.8$ & 0.28 \\
nTrips & $113.7 \pm 40.4$ & $103.3 \pm 41.9$ & 0.25 \\
Rg, $\mathbf{k m}$ & $67.0 \pm 98.8$ & $44.0 \pm 63.5$ & 0.27 \\
$\mathbf{S}$ & $3.97 \pm 0.5$ & $3.84 \pm 0.5$ & 0.26 \\
nNightTrip & $53.2 \pm 19.4$ & $46.43 \pm 19.7$ & -0.35 \\
nUniqDest & $38.2 \pm 13.0$ & $34.8 \pm 13.9$ & 0.25 \\
nHardBrake & $0.027 \pm 0.04$ & $0.022 \pm 0.02$ & 0.15 \\
nSuddenAcc & $0.039 \pm 0.05$ & $0.034 \pm 0.03$ & 0.12 \\
OverV & $0.07 \pm 0.08$ & $0.06 \pm 0.05$ & -0.15 \\
UnderV & $0.20 \pm 0.12$ & $0.23 \pm 0.15$ & -0.22 \\
avgV, $\mathbf{m} / \mathbf{s}$ & $8.03 \pm 1.87$ & $8.04 \pm 1.83$ & -0.01 \\
avgA, $\mathbf{m} / \mathbf{s}^{\mathbf{2}}$ & $2.84 \pm 0.35$ & $2.79 \pm 0.41$ & 0.15 \\
avgJ, $\mathbf{m} / \mathbf{s}^{\mathbf{3}}$ & $1.46 \pm 0.16$ & $1.39 \pm 0.20$ & 0.39 \\
\hline
\end{tabular}

${ }^{a}$ Effect sizes (Cohen's d) of 0.2 are considered small, $0.5-0.6$ are considered medium, and 0.8 are considered large

Abbreviations: TripDist average trip distance, TotalDist total travelled distance, nTrips number of trips, $R G$ radius of gyration, $S$ entropy, $n N i g h t$ Trip number of night trips, nUniqDest number of unique destinations, $n$ HardBrake number of hard brakes per mile, $n S u d d e n A c c$ number of sudden accelerations per mile, OverV overspeed, UnderV underspeed, avgV average speed, avgA average acceleration, avg J average jerk

achieved a higher F1 score and recall score, but a lower precision score. The higher precision score of the model with driving indicators is indicative of the model's lower false-positive rate (i.e. predicted preclinical $\mathrm{AD}$, but the subject did not have preclinical $A D$ ). This may be, at least partially, due to the fact that driving features reflect actual changes that are occurring on an individual basis due to the biological presence of $\mathrm{AD}$, while APOE4 are risk factors for developing AD. In addition, it is important to note that the age and $A P O E \varepsilon 4$ status model requires only two predictors as opposed to more than ten predictors in the driving behaviour model. It is, however, more invasive and less accessible because it requires APOE genotype testing.

The predictive power of the model with driving indicators was improved by including age and $A P O E \varepsilon 4$ status [21]. In fact, the predictive model with driving behaviour and age alone achieved an F1 score of 0.88 (95\% CI 0.86-0.91), and the model with driving behaviour, age, and $A P O E \& 4$ status achieved an F1 score of 0.91 (95\% CI 0.89-0.94). This improvement is unsurprising since age and $A P O E \& 4$ are among the strongest risk factors for AD [36]. Others have shown that the ability of novel
$\mathrm{AD}$ biomarkers to predict preclinical $\mathrm{AD}$ (generally indicated by abnormality of PET or CSF amyloid biomarkers) may be improved by including age and $A P O E$ $\varepsilon 4$ status in models [34, 37, 38].

Overall, although the model with driving behaviour, age, and $A P O E \& 4$ status achieved the highest performance, the model with driving indicators and age alone is the highest performing non-invasive and accessible choice. This finding is important because, given the small size and ease of installation of vehicle GPS trackers, they can be mailed to clinics and individuals, allowing widespread use in different environments (i.e. urban and rural). It is also important to note that APOE genotype testing, although invasive, is becoming more accessible through new commercial platforms such as 23 and me [39]. Therefore, GPS driving in combination with age alone or age and $A P O E$ genotype can be used as an accurate, easily implementable, and cost-effective biomarker to identify preclinical AD.

Another key finding of our study is that the importance ranking of all the features. The results confirmed that $A P O E$ \&4 status and age are the two most important features for predicting preclinical AD. Interestingly, the

Table 4 Assessment of the model performance on the test set. Values in parentheses represent 95\% confidence intervals

\begin{tabular}{lllll}
\hline Model inputs & Precision & Recall & F1 score & AUC \\
\hline Age and APOE $\varepsilon$ 4 status & $0.84(0.802-0.875)$ & $0.79(0.770-0.861)$ & $0.85(0.833-0.852)$ & $0.88(0.861-0.927)$ \\
Driving features & $0.89(0.862-0.917)$ & $0.76(0.716-0.796)$ & $0.82(0.794-0.840)$ & $0.82(0.782-0.932)$ \\
Driving features and age & $0.94(0.909-0.959)$ & $0.84(0.794-0.876)$ & $0.88(0.858-0.906)$ & $0.94(0.881-0.962)$ \\
Driving features, age, and APOE $\varepsilon$ 4 status & $0.96(0.939-0.981)$ & $0.88(0.837-0.912)$ & $0.91(0.893-0.937)$ & $0.96(0.903-0.981)$ \\
\hline
\end{tabular}




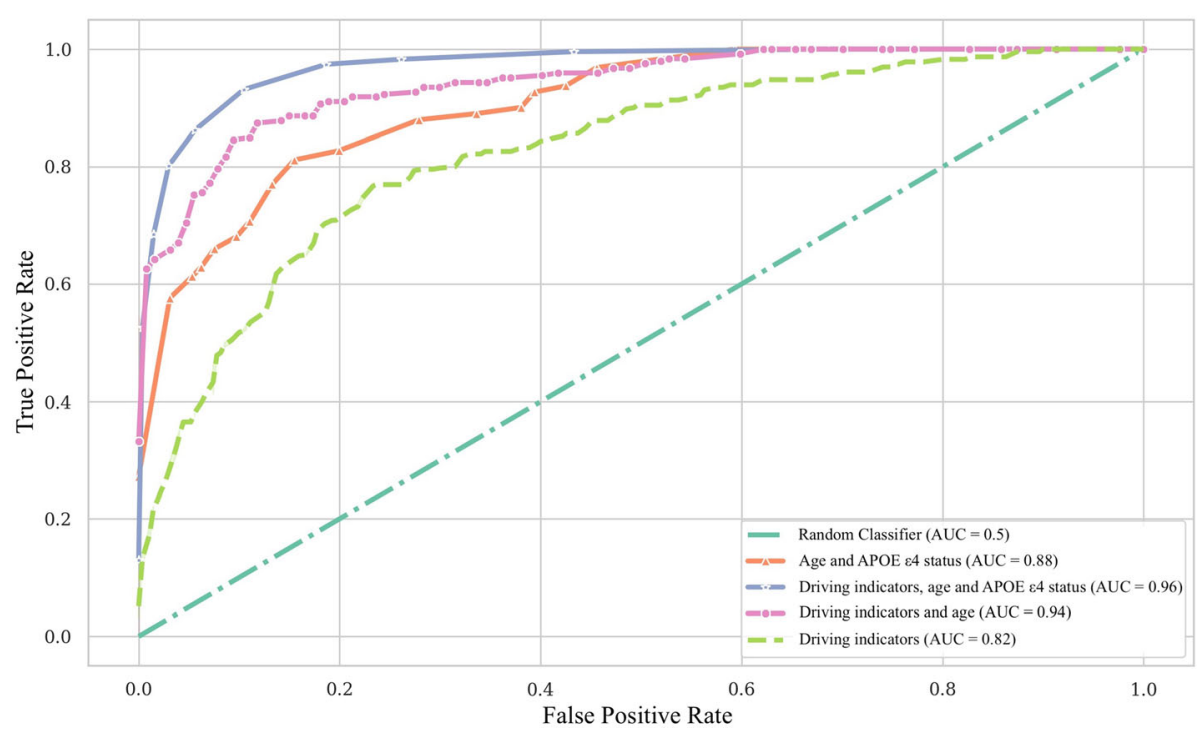

Fig. 1 The final area under the receiver operating curves (AUC) for each model. Legends show the AUC as each feature is added to the model

most important driving feature was jerk, which is a measure of the smoothness or abruptness of driving. Although studies on driving behaviour in naturalistic settings have used vehicle jerk to identify unsafe and aggressive driving behaviour [31, 40], no study to date has examined vehicle jerk to identify AD. Furthermore, the top five most important features consisted of two features describing driving performance (average jerk and over speeding) and three features describing driving space (total number of night trips, radius of gyration, and number of trips shorter than $1 \mathrm{mi}$ ). Thus, our results suggest that both driving space and driving

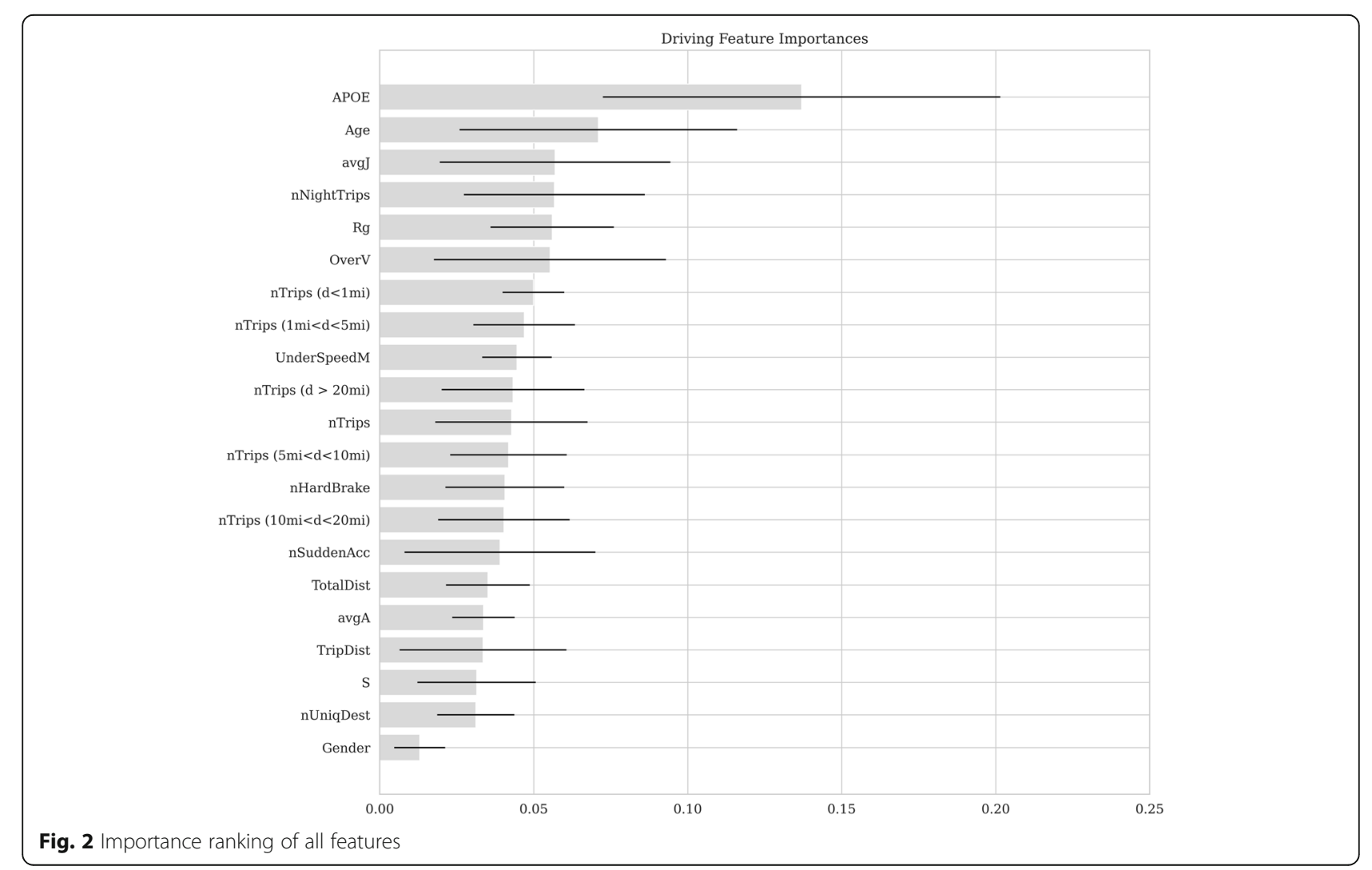


performance features have to be considered simultaneously to identify preclinical AD.

Because the decline in CSF A $\beta 42$ is one of the earliest pathological events in $\mathrm{AD}$ [41], preceding the appearance of dementia symptoms by up to 20 years, we used $A \beta 42 / A \beta 40$ as our marker of neuropathological AD among our cognitively normal participants. However, other changes in total tau and phosphorylated tau, and neurodegeneration, occur subsequently in the disease course, suggesting that future research should examine how driving behaviours predict the presence of abnormalities in other CSF biomarkers such as tau, phosphorylated tau181, phosphorylated tau217, and neurofilament light. Furthermore, recent advances in the development of plasma $\mathrm{AD}$ biomarkers have led to newly available blood tests for abnormality of AD-related proteins [42], and these blood tests may ultimately become widely used in clinical practice to diagnose AD. Machine learning methods like those used here should also be applied to determining the optimal combination of driving behaviours to identify and predict blood-based $\mathrm{AD}$ diagnoses.

\section{Limitations}

The findings of this study should be considered in light of a number of inherent limitations, which can be addressed in future work. First, although most of our participants were the sole drivers of their vehicles, no automatic method was available to identify drivers and, in fact, friends, spouses, and family members may have made a small number of trips. Second, all the participants were from the greater St. Louis metropolitan area, and thus, the findings may not be generalizable to other regions. Third, future studies with a larger sample should further investigate the role of sociodemographic attributes including sex, race, income, and education level in the driving patterns, since these attributes may contribute to various factors, including social and cultural norms affecting daily driving behaviour [43]. In addition, our sample's racial make-up, similar to that of the surrounding areas, only consists of Blacks and Whites, limiting the generalizability of the findings. Thus, future research with participants of other races and ethnicities is warranted. Finally, since repeated measures were treated as independent measures, future studies should further incorporate the longitudinal nature of the data by exploring machine learning methods that handle the correlated features from participants over time such as repeated measures random forest.

\section{Abbreviations}

AD: Alzheimer disease; ML: Machine learning; CSF: Cerebrospinal fluid; RF: Random Forest; ROC: Receiver operating curve; AUC: Area under the curve

\section{Acknowledgements}

We would like to thank all the participants who kindly took the time to participate in the study. We would like to express our gratitude to the members of Roe Lab, who contributed to data collection.

\section{Authors' contributions}

C.M.R. and S.B. designed the study. C.M.R., G.B., and S.B. contributed to the design of the framework and machine learning and statistical analyses. All authors contributed to the interpretation of the results. C.M.R. and S.B. wrote the manuscript with input from all authors. All authors provided critical feedback and helped shape the research, analysis, and manuscript. The authors read and approved the final manuscript.

\section{Funding}

Dr. Babulal is supported by the National Institute of Health/National Institute on Aging (grant numbers: AG068183, AG067428, AG056466) and by the BrightFocus Foundation (A2021142S).

Dr. Fagan has received research funding from the National Institute on Aging of the National Institutes of Health, Biogen, Centene, Fujirebio, and Roche Diagnostics. She is a member of the scientific advisory boards for Roche Diagnostics, Genentech, and AbbVie and also consults for Diadem, DiamiR, and Otsuka Pharmaceuticals. There are no conflicts.

Dr. Morris is funded by NIH grants \# P30AG066444, P01AG003991,

P01AG026276, U19AG032438, and U19AG024904. Neither Dr. Morris nor his family owns stock or has equity interest (outside of mutual funds or other externally directed accounts) in any pharmaceutical or biotechnology company.

Dr. Roe is supported by the National Institute of Health/National Institute on Aging (grant numbers: R01AG056466, R01AG068183, R01AG067428) and by the BrightFocus Foundation (A2021142S).

Dr. Schindler is supported by K23AG053426.

Availability of data and materials

The datasets used and/or analysed during the current study are available from the corresponding author on reasonable request.

\section{Declarations}

Ethics approval and consent to participate

Participants provided written informed consent, and all study procedures were approved by the Washington University Human Research Protection Office.

Consent for publication

Not applicable.

\section{Competing interests}

The authors declare that they have no competing interests.

\section{Author details}

${ }^{1}$ Institute of Biomedical Engineering, University of Toronto, 550 University Avenue, Toronto, ON M5G 1X5, Canada. ${ }^{2}$ KITE Research Institute, Toronto Rehabilitation Institute, Toronto, ON, Canada. ${ }^{3}$ Charles F. and Joanne Knight Alzheimer Disease Research Center, Washington University School of Medicine, St. Louis, MO, USA. ${ }^{4}$ Department of Neurology, Washington University School of Medicine, St. Louis, MO, USA. ${ }^{5}$ Department of Psychology, University of Johannesburg, Johannesburg, South Africa. ${ }^{6}$ Hope Center for Neurological Disorders, Washington University School of Medicine, St. Louis, MO, USA. 'Department of Pathology \& Immunology, Washington University School of Medicine, St. Louis, MO, USA. ${ }^{8}$ Department of Physical Therapy, Washington University School of Medicine, St. Louis, MO, USA. ${ }^{9}$ Department of Occupational Science \& Occupational Therapy, Washington University School of Medicine, St. Louis, MO, USA. ${ }^{10}$ Department of Occupational Science and Occupational Therapy, University of Toronto, Toronto, ON, Canada. 
Received: 6 April 2021 Accepted: 31 May 2021 Published online: 14 June 2021

\section{References}

1. World Alzheimer Report 2019: Attitudes to dementia. London: Alzheimer's Disease International; 2019. (Alzheimer's Disease International.).

2. 2018 Alzheimer's disease facts and figures. Alzheimer's \& Dementia. 2018 Mar 1;14(3):367-429.

3. Roe CM, Ances BM, Head D, Babulal GM, Stout SH, Grant EA, et al. Incident cognitive impairment: longitudinal changes in molecular, structural and cognitive biomarkers. Brain. 2018;141(11):3233-48. https://doi.org/10.1093/ brain/awy244

4. 2020 Alzheimer's disease facts and figures. Alzheimer's \& Dementia. 2020; 16(3):391-460.

5. Hill J, Fillit H, Thomas SK, Chang S. Functional impairment, healthcare costs and the prevalence of institutionalisation in patients with Alzheimer's disease and other dementias. Pharmacoeconomics. 2006;24(3):265-80. https://doi.org/10.2165/00019053-200624030-00006.

6. Deb A, Sambamoorthi U, Thornton JD, Schreurs B, Innes K. Direct medical expenditures associated with Alzheimer's and related dementias (ADRD) in a nationally representative sample of older adults - an excess cost approach. Aging Mental Health. 2018;22(5):619-24. https://doi.org/10.1 080/13607863.2017.1286454.

7. Angelucci F, Cechova K, Valis M, Kuca K, Zhang B, Hort J. MicroRNAs in Alzheimer's disease: diagnostic markers or therapeutic agents? Front Pharmacol. 2019;10. Available from: https://www.frontiersin.org/articles/10.33 89/fphar.2019.00665/full. [cited 2021 Mar 24]

8. Fish PV, Steadman D, Bayle ED, Whiting P. New approaches for the treatment of Alzheimer's disease. Bioorganic Med Chem Lett. 2019;29(2): 125-33. https://doi.org/10.1016/j.bmcl.2018.11.034.

9. O'Bryant SE, Mielke MM, Rissman RA, Lista S, Vanderstichele $H$, Zetterberg $H$, et al. Blood-based biomarkers in Alzheimer disease: current state of the science and a novel collaborative paradigm for advancing from discovery to clinic. Alzheimer's Dementia. 2017;13(1):45-58. https://doi.org/10.1016/j.jalz.2 016.09.014.

10. Handels RLH, Wimo A, Dodel R, Kramberger MG, Visser PJ, Molinuevo JL, et al. Cost-utility of using Alzheimer's disease biomarkers in cerebrospinal fluid to predict progression from mild cognitive impairment to dementia. J Alzheimer's Disease. 2017;60(4):1477-87. https://doi.org/10.3233/JAD-170324.

11. Sperling RA, Karlawish J, Johnson KA. Preclinical Alzheimer disease-the challenges ahead. Nat Rev Neurol. 2013;9(1):54-8. https://doi.org/10.1038/ nrneurol.2012.241.

12. Bature F, Guinn B, Pang D, Pappas Y. Signs and symptoms preceding the diagnosis of Alzheimer's disease: a systematic scoping review of literature from 1937 to 2016. BMJ Open. 2017;7(8):e015746. https://doi.org/10.1136/ bmjopen-2016-015746.

13. Coughlan G, Laczó J, Hort J, Minihane A-M, Hornberger M. Spatial navigation deficits - overlooked cognitive marker for preclinical Alzheimer disease? Nat Rev Neurol. 2018;14(8):496-506. https://doi.org/10.1038/s41582-018-0031-x.

14. Allison SL, Fagan AM, Morris JC, Head D. Spatial navigation in preclinical Alzheimer's disease. J Alzheimers Dis. 2016;52(1):77-90. https://doi.org/1 0.3233/JAD-150855.

15. Hird MA, Egeto P, Fischer CE, Naglie G, Schweizer TA. A systematic review and meta-analysis of on-road simulator and cognitive driving assessment in Alzheimer's disease and mild cognitive impairment. J Alzheimer's Disease. 2016:53(2):713-29. https://doi.org/10.3233/JAD-160276.

16. Eby DW, Silverstein NM, Molnar LJ, LeBlanc D, Adler G. Driving behaviors in early stage dementia: a study using in-vehicle technology. Accident Anal Prev. 2012;49:330-7. https://doi.org/10.1016/j.aap.2011.11.021.

17. Kostyniuk LP, Molnar LJ. Self-regulatory driving practices among older adults: health, age and sex effects. Accident Anal Prev. 2008;40(4):1576-80. https://doi.org/10.1016/j.aap.2008.04.005

18. Davis JD, Papandonatos GD, Miller LA, Hewitt SD, Festa EK, Heindel WC, et al. Road test and naturalistic driving performance in healthy and cognitively impaired older adults: does environment matter? J Am Geriatr Soc. 2012;60(11):2056-62. https://doi.org/10.1111/j.1532-5415.2012.04206.X.

19. Babulal GM, Stout SH, Benzinger TLS, Ott BR, Carr DB, Webb M, et al. A naturalistic study of driving behavior in older adults and preclinical Alzheimer disease: a pilot study. J Appl Gerontol. 2019;38(2):277-89. https:// doi.org/10.1177/0733464817690679.
20. Roe CM, Stout SH, Rajasekar G, Ances BM, Jones JM, Head D, et al. A 2.5year longitudinal assessment of naturalistic driving in preclinical Alzheimer's disease. J Alzheimers Dis. 2019;68(4):1625-33. https://doi.org/10.3233/JAD-1 81242.

21. Babulal GM, Johnson A, Fagan AM, Morris JC, Roe CM. Identifying preclinical Alzheimer's disease using everyday driving behavior: proof of concept. J Alzheimer's Dis. 2021;79(3):1009-14. https://doi.org/10.3233/JAD-201294.

22. Morris JC. The Clinical Dementia Rating (CDR): current version and scoring rules. Neurology. 1993;43(11):2412. https://doi.org/10.1212/wnl.43.11.2412-a.

23. Fagan AM, Mintun MA, Mach RH, Lee S-Y, Dence CS, Shah AR, et al. Inverse relation between in vivo amyloid imaging load and cerebrospinal fluid Abeta42 in humans. Ann Neurol. 2006:59(3):512-9. https://doi.org/10.1002/a na.20730.

24. Volluz K, Schindler SE, Rachel LH, Xiong C, Tammie B, Holtzman D, et al. Correspondence of CSF biomarkers measured by Lumipulse assays with amyloid PET. 2021

25. Cruchaga C, Kauwe JSK, Mayo K, Spiegel N, Bertelsen S, Nowotny P, et al. SNPs associated with cerebrospinal fluid phospho-tau levels influence rate of decline in Alzheimer's disease. Plos Genet. 2010;6(9):e1001101. https://doi. org/10.1371/journal.pgen.1001101.

26. Babulal GM, Stout SH, Head D, Holtzman DM, Fagan AM, Morris JC, et al. Neuropsychiatric symptoms and Alzheimer's disease biomarkers predict driving decline: brief report. J Alzheimer's Dis. 2017;58(3):675-80. https://doi. org/10.3233/JAD-170067

27. Bayat S, Naglie G, Rapoport MJ, Stasiulis E, Widener MJ, Mihailidis A. A GPSbased framework for understanding outdoor mobility patterns of older adults with dementia: an exploratory study. GER. 2021:1-15. https://doi. org/10.1159/000515391.

28. Bayat S, Ye B, Stasiulis E, Rapoport MJ, Naglie G, Mihailidis A. Towards a novel set of GPS-derived metrics to identify the differences between mobility patterns of cognitively intact older adults and older adults with dementia. In ALZ; 2020. Available from: https://alz.confex.com/alz/20a msterdam/meetingapp.cgi/Paper/39485. [cited 2020 Aug 26]

29. Gonzalez MC, Hidalgo CA, Barabasi A-L. Understanding individual human mobility patterns. Nature. 2008;453(7196):779-82. https://doi.org/10.1038/na ture06958.

30. Qin S-M, Verkasalo H, Mohtaschemi M, Hartonen T, Alava M. Patterns, entropy, and predictability of human mobility and life. Plos One. 2012;7(12). Available from: https://www.ncbi.nlm.nih.gov/pmc/articles/PMC3530566/. [cited 2020 Apr 29]

31. Bagdadi O, Várhelyi A. Development of a method for detecting jerks in safety critical events. Accid Analy Prev. 2013;50:83-91. https://doi.org/10.101 6/j.aap.2012.03.032.

32. Qi Y. Random Forest for Bioinformatics. In: Zhang C, Ma Y, editors. Ensemble machine learning: methods and applications Boston: Springer US; 2012. p. 307-323. doi:https://doi.org/10.1007/978-1-4419-9326-7_11, [cited 2021 Mar 25] 2012

33. Touw WG, Bayjanov JR, Overmars L, Backus L, Boekhorst J, Wels M, et al. Data mining in the Life Sciences with Random Forest: a walk in the park or lost in the jungle? Brief Bioinformatics. 2013;14(3):315-26. https://doi.org/1 0.1093/bib/bbs034.

34. Schindler SE, Bollinger JG, Ovod V, Mawuenyega KG, Li Y, Gordon BA, et al. High-precision plasma $\beta$-amyloid $42 / 40$ predicts current and future brain amyloidosis. Neurology. 2019;93(17):e1647.

35. Gaetani L, Höglund K, Parnetti L, Pujol-Calderon F, Becker B, Eusebi $P$, et al. A new enzyme-linked immunosorbent assay for neurofilament light in cerebrospinal fluid: analytical validation and clinical evaluation. Alzheimers Res Ther. 2018;10(1):8. https://doi.org/10.1186/s13195-018-033 9-1.

36. Saddiki H, Fayosse A, Cognat E, Sabia S, Engelborghs S, Wallon D, et al. Age and the association between apolipoprotein E genotype and Alzheimer disease: a cerebrospinal fluid biomarker-based case-control study. Plos Med. 2020;17(8):e1003289. https://doi.org/10.1371/journal.pmed.1003289.

37. Dayon L, Wojcik J, Núñez Galindo A, Corthésy J, Cominetti O, Oikonomidi A, et al. Plasma proteomic profiles of cerebrospinal fluid-defined Alzheimer's disease pathology in older adults. J Alzheimer's Dis. 2017;60(4):1641-52. https://doi.org/10.3233/JAD-170426.

38. Pérez-Grijalba V, Arbizu J, Romero J, Prieto E, Pesini P, Sarasa L, et al. Plasma A $442 / 40$ ratio alone or combined with FDG-PET can accurately predict amyloid-PET positivity: a cross-sectional analysis from the AB255 Study. Alz Res Ther. 2019;11(1):96. https://doi.org/10.1186/s13195-019-0549-1. 
39. 23andMe. DNA genetic testing \& analysis - 23andMe Canada. Available from: https://www.23andme.com/en-ca/. [cited 2021 Mar 26]

40. Feng F, Bao S, Sayer JR, Flannagan C, Manser M, Wunderlich R. Can vehicle longitudinal jerk be used to identify aggressive drivers? An examination using naturalistic driving data. Accid Anal Prev. 2017;104:125-36. https://doi. org/10.1016/j.aap.2017.04.012

41. Jack CR, Knopman DS, Jagust WJ, Shaw LM, Aisen PS, Weiner MW, et al. Hypothetical model of dynamic biomarkers of the Alzheimer's pathological cascade. Lancet Neurol. 2010;9(1):119-28. https://doi.org/10.1016/S14 74-4422(09)70299-6.

42. Zetterberg H, Blennow K. Moving fluid biomarkers for Alzheimer's disease from research tools to routine clinical diagnostics. Mol Neurodegeneration. 2021;16(1):10. https://doi.org/10.1186/s13024-021-00430-x

43. Vaughn MG, Define RS, DeLisi M, Perron BE, Beaver KM, Fu Q, et al. Sociodemographic, behavioral, and substance use correlates of reckless driving in the United States: findings from a national sample. J Psychiatr Res. 2011;45(3):347-53. https://doi.org/10.1016/j.jpsychires.2010. 06.016.

\section{Publisher's Note}

Springer Nature remains neutral with regard to jurisdictional claims in published maps and institutional affiliations.

Ready to submit your research? Choose BMC and benefit from:

- fast, convenient online submission

- thorough peer review by experienced researchers in your field

- rapid publication on acceptance

- support for research data, including large and complex data types

- gold Open Access which fosters wider collaboration and increased citations

- maximum visibility for your research: over $100 \mathrm{M}$ website views per year

At BMC, research is always in progress.

Learn more biomedcentral.com/submissions 